\title{
MP-AzeFlu provides rapid and effective allergic rhinitis control: results of a non-interventional study in Romania*
}

\author{
Ioana Agache1, Ion-Caius Doros², Polliana Mihaela Leru³, , Irina Bucur ${ }^{5}$, \\ Marioara Poenaru², Codrut Sarafoleanu ${ }^{3,6}$ \\ 'Transylvania University of Brasov, Faculty of Medicine, Department of Allergy \& Clinical Immunology, Brasov, Romania \\ 2 University of Medicine and Pharmacy Victor Babes, ENT Department, Timisoara, Romania \\ ${ }^{3}$ Carol Davila University of Medicine and Pharmacy, Family Medicine Department, Bucharest, Romania \\ ${ }^{4}$ Colentina Clinical Hospital, Internal Medicine Department, Bucharest, Romania \\ ${ }^{5}$ Nicolae Malaxa Hospital, Allergology Outpatient Unit, Bucharest, Romania \\ ${ }^{6}$ Santa Maria Hospital, University of Medicine and Pharmacy, Department of ORL \& HNS, Bucharest, Romania
}

Rhinology 56: 33-41, 2018

https://doi.org/10.4193/Rhino16.278

*Received for publication:

September 26, 2016

Accepted: August 19, 2017

\begin{abstract}
Background: Allergic Rhinitis and its Impact on Asthma (ARIA) and the European Union (EU) recommend a shift to guide allergic rhinitis (AR) treatment decisions from symptom severity to disease control, using a simple visual analogue scale (VAS). Using this VAS we assessed, in a real-life study in Romania, the effectiveness of MP-AzeFlu nasal spray.
\end{abstract}

Methodology: In this multi-centre, prospective, non-interventional study, 253 patients ( $\geq 12$ years old) with moderate-to-severe AR were prescribed MP-AzeFlu and assessed their symptoms on a VAS ( 0 (not at all bothersome) to $100 \mathrm{~mm}$ (very bothersome)) on Days 0, 1, 3, 7 and $\sim 14$. The proportion of patients who achieved a defined VAS score cut-off for well-controlled (38 mm) AR were also calculated. Patients' perception of disease control was assessed on Day 3.

Results: MP-AzeFlu use was associated with a mean (standard deviation) VAS score reduction from 78.4 (15.1) mm at baseline to 14.7 (15.1) $\mathrm{mm}$ on the last day. Effectiveness was consistent irrespective of disease severity, phenotype or patient age. $83.4 \%$ of patients achieved the $\leq 38 \mathrm{~mm}$ 'well-controlled'VAS score cut-off by last day and $95.2 \%$ considered their symptoms to be well- or partly controlled at Day 3.

Conclusions: MP-AzeFlu provided rapid, effective and sustained AR symptom control in a real-life setting in Romania, irrespective of severity, phenotype or patient age, aligning with ARIA and EU recommendations and supporting MP-AzeFlu's position as the drug of choice for the treatment of moderate-to-severe AR.

Key words: azelastine, control, effectiveness, fluticasone propionate, visual analogue scale

\section{Introduction}

Allergic rhinitis (AR) is a global health problem, the most common chronic disease affecting citizens today worldwide. Up-to-date AR prevalence data in Romania are lacking ${ }^{(1)}$, but data from 1998 showed a prevalence of 11.4-15.0\% among children aged 13-14 years, and the prevalence is rising ${ }^{(2-4)}$. When severe symptoms are present patients suffer from a negative impact on their day-to-day lives and quality of life (QoL), as well as detrimental effects on sleep, mood, cognitive functioning, and social inter-relationships ${ }^{(5-9)}$. The socio economic cost of AR is also high, driven primarily by high rates of presenteeism ${ }^{(10,11)}$.
Intranasal corticosteroids (INS) are considered the most effective AR medication class ${ }^{(12,13)}$. However, many patients are dissatisfied with therapy ${ }^{(14)}$, remain symptomatic ${ }^{(10)}$, with high rates of poly-pharmacy reported ${ }^{(15,16)}$; a practice neither endorsed by the guidelines ${ }^{(12,13)}$ nor supported by the evidence ${ }^{(17,18)}$. There is a clear need for more effective AR therapies which provide more rapid and complete symptom control.

Two recent developments in the field of AR bring us closer to achieving that goal. The first of these has been the MACVIA-ARIA (Contre les Maladies Chroniques pour un Vieillissement Actif- 
Allergic Rhinitis and its Impact on Asthma)- and EU-endorsed shift in the focus of AR management from reduction in symptom severity to achievement of disease control ${ }^{(19)}$. A simple visual analogue scale (VAS) has been adopted as the new language of AR disease control ${ }^{(20)}$ and embedded into an updated MACVIAARIA treatment guideline (the AR clinical decision support system (CDSS)) ${ }^{(21)}$. Within this guideline, a VAS score cut-off of $5 / 10 \mathrm{~mm}$ is used both to assess control and guide treatment decisions ${ }^{(21)}$. This same VAS has also been incorporated into an AR control app for patients (Allergy Diary) ${ }^{(22)}$, with the companion app for physicians currently under development, empowering patients to take control of their own AR, facilitating improved patient-physician communication and encouraging guidelinedirected care.

The second development has been the introduction of MPAzeFlu, (Dymista ${ }^{\oplus}$, Meda, Solna, Sweden), a new class of AR medication (ATC R01AD58) comprising a novel formulation of fluticasone propionate (FP), azelastine hydrochloride and excipients delivered in a single intranasal spray. In a randomized controlled trial (RCT) setting MP-AzeFlu provided twice the overall nasal and ocular symptom relief as an INS (23). Moreover, one in six patients with moderate-to-severe seasonal AR (SAR) and seven in ten patients with mild-to-moderate perennial AR (PAR) achieved complete or near-to-complete symptom relief on MP-AzeFlu, and did so about a week faster than those treated with FP ${ }^{(23,24)}$.

Undoubtedly, RCTs remain the gold standard trial design for the assessment of efficacy, by establishing a clear cause and effect relationship between treatment and outcome. However, by virtue of their strict inclusion/exclusion criteria they rarely reflect the heterogeneity of real-world patients and primary care populations ${ }^{(25)}$. Data generated from well-designed real-life studies are generalizable to a broad patient population and provide evidence supplementary to that obtained from RCTs, helping to translate guideline recommendations into health policies ${ }^{(26)}$.

The generalisability of MP-AzeFlu data derived from clinical studies to the Romanian population is unknown. The aim of the present non-interventional study was to assess the effectiveness of MP-AzeFlu in achieving AR control in real-life clinical practice in Romania, using the VAS, in line with MACVIA-ARIA and EU recommendations.

\section{Materials and methods}

Study design

The primary objectives of the study were to gather knowledge on use of MP-AzeFlu in routine clinical practice in Romania and to assess its effectiveness in a real-life setting using a VAS. This was a multicentre, prospective, non-interventional study conducted in Romania between July 2014 and August 2015, and was part of a larger Europe-wide study of similar design. Patients were prescribed MP-AzeFlu (1 spray/nostril bid) at the inclusion visit and instructed to use it for 14 days, with some flexibility in duration to allow for routine clinical practice. Patients recorded effectiveness of MP-AzeFlu on a diary card, and returned this card at a follow-up visit or by mail. There were no restrictions regarding concomitant treatments, except for ritonavir which should be avoided.

The study was carried out in accordance with current Romanian laws and guidelines, which respect and reflect European guidelines on study conduct. The study design was approved by the Romanian National Ethics Committee.

\section{Inclusion/exclusion criteria}

Patients were eligible to enter this study if they were aged 12 years or older, had moderate-to-severe SAR or PAR (ARIAdefined) ${ }^{(12)}$, and were prescribed MP-AzeFlu according to its approved indication in Romania (i.e. if monotherapy with either intranasal antihistamine or glucocorticoid was not considered sufficient) ${ }^{(27)}$. Patients were required to have acute AR symptoms on the day of inclusion, defined as a recommended VAS score $>$ $50 \mathrm{~mm}$. However, patients could be enrolled into the study regardless of their VAS score if the physician rated their symptoms as moderate-to-severe. Patients were excluded if they had hypersensitivity to azelastine hydrochloride, FP or to any of the excipients. Female patients were excluded if they were pregnant or breast-feeding. All patients provided written informed consent, and if below 18 years of age, their caregiver also provided signed consent.

\section{Physicians}

Practicing physicians from Romania recruited patients into this study. These were physicians usually involved in AR management and included allergists, ear, nose and throat (ENT) specialists and general practitioners (GP). The physician's decision to include a patient in the study was made independently from, and after, the decision to prescribe MP-AzeFlu, and in accordance with the current summary of product characteristics for MP-AzeFlu ${ }^{(27)}$. Each physician could enrol 10-15 patients into the study.

\section{Allergic rhinitis classification}

In this study, SAR was defined as allergy (history and specific $\operatorname{lgE}$ ) to at least one pollen allergen (i.e. spring, summer and/or autumn pollen; but no non-pollen allergens). PAR was defined as allergy to at least one non-pollen allergen (i.e. dust mites, pet dander and/or mould) but no pollen allergens. Patients with SAR + PAR were those defined as having allergy to at least one pollen and at least one non-pollen allergen. AR of unknown origin was 
defined as allergy to other or unknown allergens (i.e. not one of the allergens listed above) or unknown triggers (i.e. rhinitis supported by history but not specific IgE data). Some of these patients may have had non-allergic rhinitis. Given the fact that in real life (especially in primary care), therapeutic decisions are made without any evidence of specific lgE data this subgroup is of particular importance to evaluate the effectiveness of the therapeutic intervention.

Patients with intermittent AR were defined as those who suffered from AR symptoms for $\leq 4$ days/week OR for $\leq 4$ consecutive weeks. Persistent AR was diagnosed when patients suffered from AR symptoms for $>4$ days/week AND for $>4$ consecutive weeks.

\section{Data collection and assessments}

At the inclusion visit physicians recorded information on patient demographics, clinical symptoms, and previous AR treatments. They also recorded AR history, number of visits in the current calendar year due to $A R$, predominant symptoms of $A R$ and documented ARIA-defined AR severity. The reason for patients' visit ("acute AR symptoms," "expected allergen exposure in near future" or "other") and the reason for prescribing MP-AzeFlu ("other therapies were not sufficient in the past," "other therapies were not considered to be sufficient to treat acute symptoms," or "other") were also recorded. All data were recorded by physicians in an electronic case report form (eCRF; Trium Analysis Online $\mathrm{GmbH}$ ) set up in English language.

\section{MP-AzeFlu effectiveness assessment}

Patients assessed AR symptom severity in the morning prior to MP-AzeFlu use on Days 0, 1, 3, 7 and the last day using a VAS ranging from $0 \mathrm{~mm}$ (not at all bothersome) to $100 \mathrm{~mm}$ (very bothersome) in response to the statement "please reflect on how bothersome your symptoms were within the previous 24 hours." Patient-perceived level of disease control within the previous 24 hours in the morning on Day 3 was recorded on a patient card as 'well-controlled,' partly controlled' or 'uncontrolled.'

\section{Safety}

All suspected adverse drug reactions (ADRs) and special situations (i.e. pregnancy; breastfeeding; any overdose, abuse, offlabel use, misuse or medication error; adverse reaction related to occupational exposure; lack of efficacy) were recorded by the physician. An ADR was defined as an adverse event (AE) with a reasonable possibility that the $A E$ may have been linked to MP-AzeFlu. All AEs were coded using the Medical Dictionary for Regulatory Activities (MedDRA) coding system (Version 18.0).

\section{Statistics}

This study included 253 patients, which was considered suf-
Table 1. Patient characteristics.

\begin{tabular}{|c|c|}
\hline Characteristic & n (\%) \\
\hline \multicolumn{2}{|l|}{ Gender } \\
\hline Female & $138(54.6)$ \\
\hline \multicolumn{2}{|l|}{ Age, yr } \\
\hline $12-17$ & $13(5.1)$ \\
\hline $18-65$ & $230(90.9)$ \\
\hline$>65$ & $10(4.0)$ \\
\hline Duration of rhinitis, ${ }^{*}$ year (mean [SD]) & $21.6(11.5)$ \\
\hline \multicolumn{2}{|l|}{ Type of rhinitis (Traditional classification) } \\
\hline SAR & $55(21.7)$ \\
\hline PAR & $50(19.8)$ \\
\hline $\mathrm{SAR}+\mathrm{PAR}$ & $104(41.1)$ \\
\hline Unknown origin & $44(17.4)$ \\
\hline \multicolumn{2}{|l|}{ Type of rhinitis (ARIA classification) } \\
\hline Intermittent AR & $57(22.5)$ \\
\hline Persistent AR & $184(72.7)$ \\
\hline Unknown AR pattern & $10(4.0)$ \\
\hline \multicolumn{2}{|l|}{ Severity of $A R^{* *}$} \\
\hline Troublesome symptoms & $215(85.0)$ \\
\hline Impairment of daily activities/leisure/sport & $187(73.9)$ \\
\hline Impairment of school/work & $139(54.9)$ \\
\hline Sleep disturbance & $113(44.7)$ \\
\hline At least one criterion & $253(100)$ \\
\hline \multicolumn{2}{|l|}{ Predominant symptoms } \\
\hline Nasal congestion & $151(59.7)$ \\
\hline Rhinorrhoea & $56(22.1)$ \\
\hline Sneezing & $27(10.8)$ \\
\hline Nasal pruritus & $19(7.5)$ \\
\hline Concomitant ocular symptoms & $152(60.1)$ \\
\hline
\end{tabular}

${ }^{*} \mathrm{n}=186$; ${ }^{*}$ moderate/severe $A R$ if at least one criterion met AR: allergic rhinitis; PAR: perennial allergic rhinitis; SAR: seasonal allergic rhinitis; SD: standard deviation.

ficient to provide insight into the effectiveness of MP-AzeFlu in real-life clinical practice in Romania. The baseline and efficacy analyses were conducted on the safety population, defined as all patients who were treated at least once with MP-AzeFlu, and whose physician had provided an electronic signature to confirm data accuracy. All data were reported using descriptive statistics, with analyses performed by the Contract Research Organisation, Syneed Medidata GmbH, using SAS Version 9.3. 

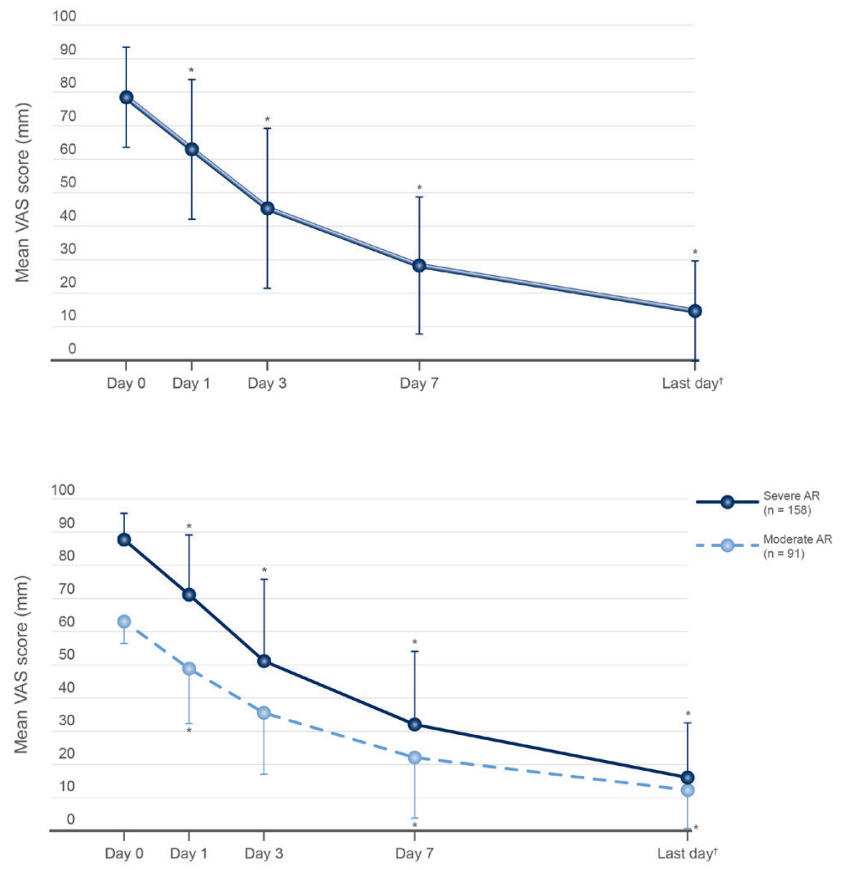

Figure 1. Effect of MP-AzeFlu on visual analogue scale (VAS) score over time in (A) the total population ( $n=253$ ) and (B) according to baseline severity in patients with ARIA-defined moderate-to-severe allergic rhinitis (AR) in routine clinical practice in Romania. Severity was categorized according to baseline VAS scores (moderate AR: baseline VAS score $=50-74 \mathrm{~mm}$; severe AR: baseline VAS score $=75-100 \mathrm{~mm}$. Data are presented as mean and standard deviation. ARIA $=$ Allergic Rhinitis and its Impact on Asthma. ${ }^{*} p<0.0001$ vs. baseline; tmean of last day corresponds to Day 15.9 .

The mean VAS scores on Days 0, 1, 3, 7 and last day were calculated for the total population $(n=253)$ and also according to baseline AR severity. Patients with baseline VAS of $50-74 \mathrm{~mm}$ ( $n=91$ ) were considered to have moderate AR; those with a baseline VAS of $75-100 \mathrm{~mm}(n=158)$ were considered to have severe AR. Mean VAS scores were also calculated for each AR phenotype on each day (SAR $(n=55)$, PAR $(n=50)$, SAR + PAR $(n=104)$, unknown $(n=44)$, intermittent $(n=57)$, persistent $(n$ $=184)$ ), and patient age group (i.e. 12-17 years $(n=13), 18-65$ years $(n=230)$, $>65$ years $(n=10))$. Change in VAS score from baseline to Days 1, 3, 7 and last day was assessed as contrast from repeated measurement ANCOVA. The covariance matrix was left unspecified. A p-value $<0.05$ was judged to be significant. Patients' perception of symptom control was calculated for the total population and subdivided by phenotype.

\section{Post-hoc analyses}

A weighted mean of country-specific VAS score cut-offs (Youden index) to define 'well-controlled' and 'partly controlled' AR on Day 3 were calculated from a pooled data set incorporating data from Germany, Sweden, Denmark, Norway and Romania ${ }^{(28)}$ and
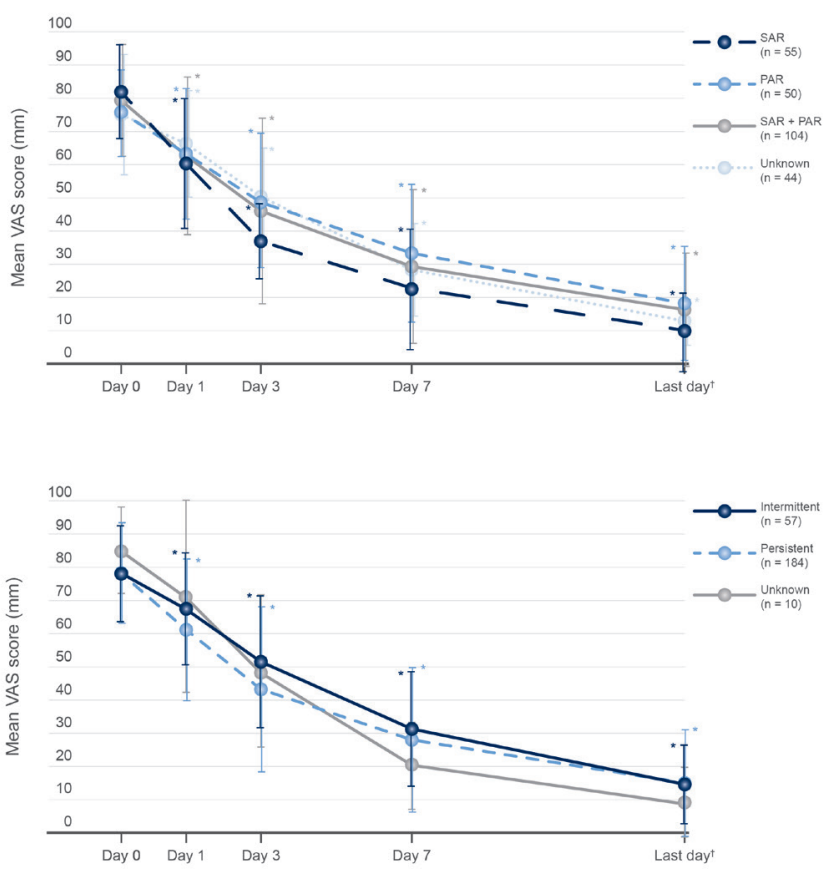

Figure 2. Effect of MP-AzeFlu on visual analogue scale (VAS) score over time according to $(A)$ allergic rhinitis phenotype (SAR, PAR, both or unknown) and (B) ARIA-defined phenotype (intermittent or persistent AR or unknown) in patients with ARIA-defined moderate-to-severe allergic rhinitis in routine clinical practice in Romania. $A R=$ allergic rhinitis, ARIA = Allergic Rhinitis and its Impact on Asthma, PAR = perennial allergic rhinitis, $S A R=$ seasonal allergic rhinitis. Data are presented as mean and standard deviation. ${ }^{*} p<0.0001$ vs. baseline; tmean of last day corresponds to Day 15.9 .

were $38 \mathrm{~mm}$ and $55 \mathrm{~mm}$, respectively. Response was defined as achievement of at least these cut-offs on Days 0, 1, 3, 7 and last day. Responder rates were derived from time to response analysis as Kaplan-Meier estimates. Time at which patients achieved the CDSS-defined AR control VAS score threshold (i.e. $50 \mathrm{~mm})^{(21)}$ was also assessed.

\section{Results}

\section{Patient disposition}

Overall, 20 Romanian physicians enrolled 255 patients into this study. Two patients were excluded from the analysis due to unconfirmed data documentation (i.e. missing physician signatures). Therefore, the safety population comprised 253 patients.

\section{Patient demographic and baseline characteristics} Patient demographics and baseline clinical characteristics are presented in Table 1. The majority of the population were adults aged $18-65$ years $(n=230 ; 90.9 \%)$ and a slightly greater proportion of the population was female $(n=138 ; 54.5 \%)$. About two-thirds of patients had SAR, either alone $(n=55 ; 21.7 \%)$ or in combination with PAR $(n=104 ; 41.1 \%)$. Only 50 patients $(19.8 \%)$ 


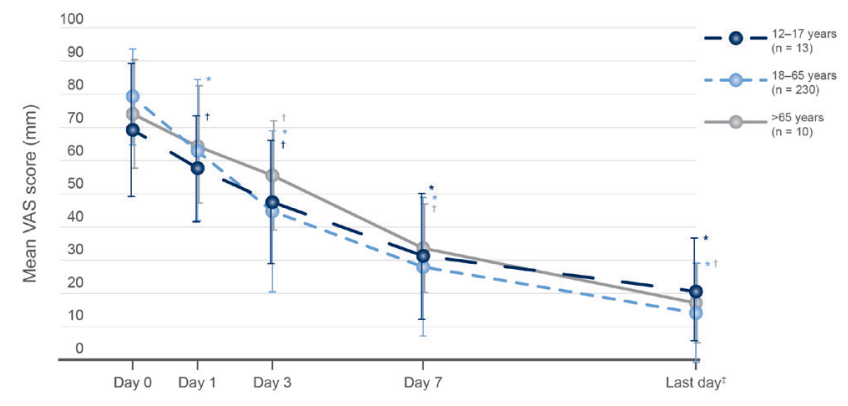

Figure 3. Effect of MP-AzeFlu on visual analogue scale (VAS) score over time according to patient age in patients with ARIA-defined moderateto-severe allergic rhinitis in routine clinical practice in Romania. Data are presented as mean and standard deviation. ARIA = Allergic Rhinitis and its Impact on Asthma. ${ }^{*} p<0.0001$ vs. baseline; $\uparrow p \leq 0.0164$ vs. baseline; $\ddagger$ mean of last day corresponds to Day 15.9 .

had PAR alone and $17.4 \%(n=44)$ had AR of unknown origin. $72.7 \%$ ( $n=184$ ) were classified as having persistent AR (i.e. symptoms for $\geq 4$ days/week AND for $\geq 4$ consecutive weeks) ${ }^{(12)}$. In total, 249 patients (98.4\%) had a baseline VAS score $>50 \mathrm{~mm}$. As anticipated based on inclusion criteria, $100 \%$ of patients had moderate-to-severe AR, according to the ARIA classification ${ }^{(12)}$. Only 4 of 253 patients had a baseline VAS score $<50 \mathrm{~mm}$. The mean (SD) duration of AR was 21.6 (11.5) years. Nasal congestion was the predominant AR symptom ( $n=151 ; 59.7 \%) .60 .1 \%$ of patients $(n=152)$ had concomitant ocular symptoms.

\section{Physician visits}

In the current calendar year, the mean (SD) number of physician visits due to AR was 1.4 (1.6). 62.1\% ( $n=157)$ of patients had visited their physician at least once before inclusion into the study in the current calendar year due to their AR; $25.7 \%$ ( $n=$ $65)$ of patients had attended once before, $16.2 \%(n=41)$ had attended twice before, $7.5 \%(n=19)$ three times before, $7.1 \%(n$ $=18$ ) four times before and $5.5 \%(n=14)$ had made five or more visits, prior to the current visit. The most common reasons for the physician visit were 'acute AR symptoms' ( $n=224 ; 88.5 \%)$, 'expected allergen exposure in the near future' $(n=39 ; 15.4 \%)$ and 'other' $(n=22 ; 8.7 \%)$. The most frequent reason for prescribing MP-AzeFlu was that 'other therapies were not sufficient in the past' $(n=192 ; 75.9 \%)$.

\section{AR treatments in previous year}

The most commonly used AR medications in the last year were oral antihistamines ( $n=217 ; 85.8 \%)$, oral/intranasal decongestants ( $n=200 ; 79.1 \%$ ) and INS ( $n=177 ; 70.0 \%$ ) (Table 2). Eye drops, either antihistamine or mast cell stabilizer were used by 34 patients (13.4\%). Overall, 215 patients (85.0\%) had used multiple AR treatments in the last year. Nineteen patients (7.5\%) had undergone previous immunotherapy and a further 11 (4.3\%)
Table 2. AR treatment in the study group.

\begin{tabular}{|lc|}
\hline AR treatment & n (\%) \\
\hline Oral antihistamine & $217(85.8)$ \\
\hline Intranasal corticosteroid & $177(70.0)$ \\
\hline Intranasal decongestant & $152(60.1)$ \\
\hline Oral decongestant & $48(19.0)$ \\
\hline Intranasal antihistamine & $30(11.9)$ \\
\hline Oral leukotriene receptor antagonist & $27(10.7)$ \\
\hline Ocular antihistamine & $25(9.9)$ \\
\hline Systemic corticosteroid & $22(8.7)$ \\
\hline Ocular mast cell stabiliser & $9(3.6)$ \\
\hline Intranasal mast cell stabiliser & $5(2.0)$ \\
\hline Other & $2(0.8)$ \\
\hline None & $5(2.0)$ \\
\hline Unknown & $1(0.4)$ \\
\hline Immunotherapy (in the past or ongoing) & $30(11.9)$ \\
\hline Number of medications listed above (excluding immunotherapy) & $38(15.0)$ \\
\hline 1 & $79(31.2)$ \\
\hline 2 & $69(27.3)$ \\
\hline 3 & $37(14.6)$ \\
\hline 5 & $18(7.1)$ \\
\hline 7 & $7(2.8)$ \\
\hline & $4(1.6)$ \\
\hline & $1(0.4)$ \\
\hline
\end{tabular}

AR: allergic rhinitis

were undergoing immunotherapy at the time of the inclusion visit.

\section{Effectiveness}

The mean (SD) time period between commencing MP-AzeFlu treatment and the VAS assessment on the last visit (or the day the patient returned his or her card) was 15.9 (4.7) days (median 14 days; Q1, 14 days; Q3, 15 days).

\section{A. VAS score}

Under MP-AzeFlu treatment the mean (SD) VAS score decreased from $78.4(15.1) \mathrm{mm}$ at baseline to $14.7(15.1) \mathrm{mm}$ at the last visit, corresponding to a mean (SD) reduction of $63.6(20.4) \mathrm{mm}$ (Figure 1A). The treatment effect was fast, sustained and independent of disease severity (Figure 1B) or phenotype (Figure $2 \mathrm{~A}$ and Figure $2 \mathrm{~B}$ ). Patients with SAR, PAR, both SAR and PAR, or 


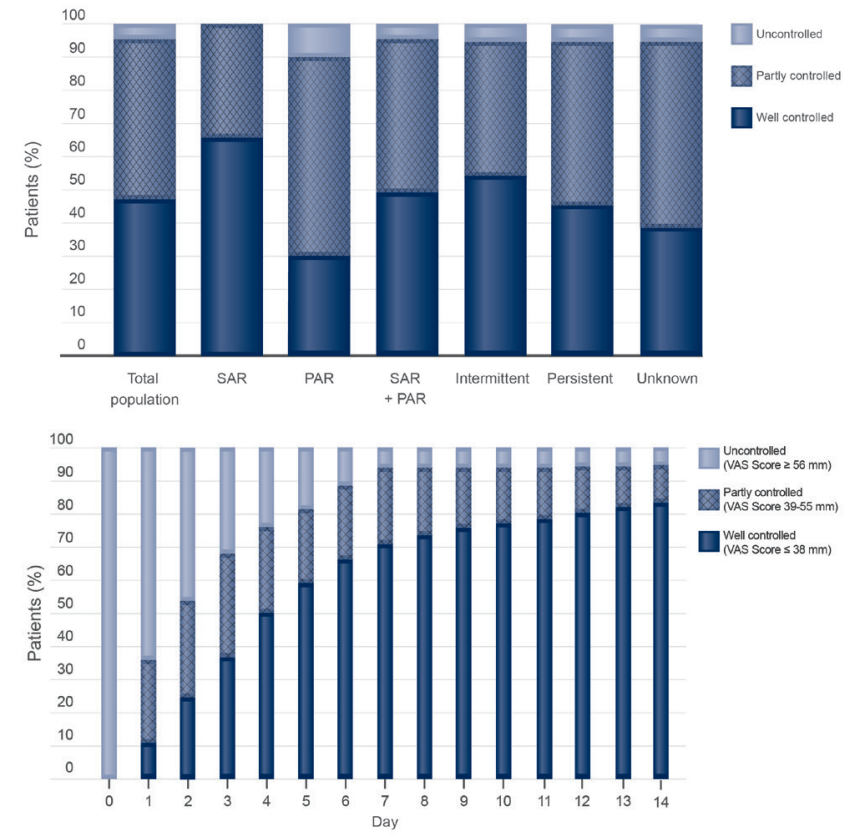

Figure 4. (A) Patient perception of AR control following 3 days' treatment with MP-AzeFlu and (B) proportion of patients treated with MP-AzeFlu who had well-controlled (defined as VAS score $\leq 38 \mathrm{~mm}$ ), partly controlled (VAS score $39-55 \mathrm{~mm}$ ) and uncontrolled (VAS score $\geq$ $56 \mathrm{~mm}$ ) allergic rhinitis over time. Patients had ARIA-defined moderateto-severe allergic rhinitis and were attending routine clinical practice in Romania. (A) Results are summarized for the total population $(n=253)$ and according to $A R$ phenotype (i.e. seasonal $A R(S A R ; n=55)$, perennial AR (PAR; $n=50)$, SAR + PAR $(n=104)$, intermittent $(n=57)$, persistent ( $n$ $=184)$, unknown $(n=44)$ ). (B) Data are presented as Kaplan Meier estimates on Days 1, 3, 7 and last day and interpolated on other days. AR = allergic rhinitis, VAS = visual analogue scale.

AR of unknown origin, and those with intermittent or persistent disease experienced rapid and clinically relevant symptom control on MP-AzeFlu. The effectiveness of MP-AzeFlu was also independent of patient age, with a similar reduction in VAS score observed for adolescents, adults and the elderly (Figure 3 ).

\section{B. Disease control as perceived by the patients}

MP-AzeFlu treatment was associated with a rapid reduction in AR symptom burden as perceived by the patient; three days after the start of treatment, $47.0 \%(n=119)$ of patients considered their symptoms well-controlled and $48.2 \%(n=122)$ rated their symptoms as partly controlled (Figure 4 ). Only $4.7 \%$ of patients $(n=12)$ indicated that their symptoms were uncontrolled at Day 3. Over $90 \%$ of patients with PAR and over $95 \%$ of patients with SAR, SAR + PAR, AR of unknown origin and those with either intermittent or persistent disease reported their symptoms were well- or partly controlled after three days of treatment with MPAzeFlu (Figure 4A).

\section{Disease control as evaluated by the VAS score cut-offs}

The perception of 'well-controlled' symptoms corresponded to a VAS score cut-off of $38 \mathrm{~mm}$ (28). $10.9 \%$ of AR patients achieved at least this cut-off on Day 1, 36.8\% on Day 3, 70.9\% on Day 7 and $83.4 \%$ on the last day. Similarly, the feeling of 'partly controlled' symptoms corresponded to a VAS score cut-off of $55 \mathrm{~mm}$ (28). $36.0 \%, 68.0 \%, 93.9 \%$ and $94.7 \%$ of patients achieved at least this cut-off on Days 1, 3, 7 and last day, respectively. A similar response was seen for intermittent or persistent AR. A total of $50.8 \%$ of patients treated with MP-AzeFlu achieved the AR CDSS control cut-off (i.e. $50 \mathrm{~mm}$ ) on Day 3, increasing to $85.7 \%$ on Day 7 and $97.2 \%$ by Day 14 .

Safety

Three patients (1.2\%) reported a total of 4 AEs during this study, namely daytime sedation, sneezing, exacerbation of nasal blockage and nasal irritation. Nasal irritation was considered highly likely to be related to MP-AzeFlu treatment, with the other AEs considered possibly related to treatment. None of these AEs were considered serious. The patient with an exacerbation of nasal blockage and nasal irritation discontinued treatment with MP-AzeFlu due to lack of efficacy.

\section{Discussion}

There are several strengths of this well-designed non-interventional study. Firstly, it provides valuable information on the type of AR patients (severity and treatment) presenting to physicians in Romania, contributing with valuable insights into the present AR landscape in the absence of recent epidemiological data. Secondly, the results highlight the high symptomatic burden associated with AR in Romania. Thirdly, this is the first study to assess the use, effectiveness and safety of MP-AzeFlu in a real-life clinical setting in Romania, and the first to use the MACVA-ARIAand EU-endorsed evaluation of AR control (i.e. VAS). We also contextualized the VAS score results obtained with MP-AzeFlu in real life within the framework of the recently published updated ARIA guideline (i.e. AR CDSS) ${ }^{(21)}$.

The main strength of this study was the fact that it was conducted in real-life, designed to answer different questions than those posed by RCTs, and to examine interventions using more realistic, and arguably more relevant, levels of clinical care. In their daily practice, physicians see a heterogeneous AR patient population. For example, patients frequently present with comorbid conditions, with lifestyle habits affecting their health (e.g. smoking), or who are poorly concordant with their AR treatment regimen. Real-life studies help to extend the evidence base and improve applicability of findings to real-life clinical practice by showing the treatment effects that patients may expect to achieve in routine clinical care. 
Patients treated with MP-AzeFlu experienced rapid and sustained symptom control, with a consistent response noted irrespective of disease severity or phenotype, whether the phenotype was defined according to the symptom-triggering allergen (i.e. SAR/PAR) or by symptom duration (i.e. intermittent/persistent). Adolescents, adults and elderly patients equally benefited from MP-AzeFlu. Over $95 \%$ of patients felt their AR was well- or partly controlled after just 3 days and 8 of 10 patients achieved the patient-defined well-controlled VAS score cut-off (i.e. $\leq 38$ $\mathrm{mm}$ ) by last day of MP-AzeFlu treatment. $97.2 \%$ of patients treated with MP-AzeFlu achieved the AR CDSS control cut-off (i.e. 50 $\mathrm{mm})(21)$ by the last day of MP-AzeFlu treatment.

Over $60 \%$ of AR patients attending during routine clinical practice in Romania (and considered for inclusion in this trial) had SAR, with most of these also presenting with PAR (41.1\%). Over $70 \%$ of patients had persistent disease, highlighting the need for an effective and safe AR treatment that can be used for short bursts of treatment to achieve fast symptom control, as well as for long-term use. Nasal congestion was reported as the most bothersome nasal symptom, in agreement with other studies (29), and over $60 \%$ of patients complained of ocular symptoms, thought to have the greatest negative impact on patients' QoL ${ }^{(6)}$. MP-AzeFlu is a treatment choice for these patients, indicated for both SAR and PAR ${ }^{(27)}$, with rapid and sustained benefits observed in those with persistent disease ${ }^{(24)}$, proven to be three times more effective than an INS in congestion-predominant patients and over twice as effective in relieving ocular symptoms associated with $\mathrm{AR}^{(23)}$.

Patients were treated for 2 weeks only irrespective of their phenotype (SAR, PAR, SAR + PAR) or severity, based on previous studies having shown a rapid effect of MP-AzeFlu ${ }^{(24)}$. Whether this rapid effect is sustained for those with persistent disease is the subject of a currently ongoing study of 6 weeks' duration, the results of which shall be published in full.

The burden of AR was also highlighted by this study. The mean VAS score at baseline was $78.4 \mathrm{~mm}$, showing not only the high symptomatic burden experienced by these patients, but also the inadequate symptom relief provided by previously used AR treatments. The latter was confirmed by the high degree of polypharmacy noted (85.0\%), higher that that reported in other countries ${ }^{(15,30-34)}$, and the fact that over three-quarters of physicians prescribed MP-AzeFlu as other AR therapies were not sufficient in the past. Interestingly, almost $30 \%$ of physicians prescribed MP-AzeFlu first line, when they considered that other therapies would be insufficient to treat acute symptoms. Over $60 \%$ of patients had made multiple visits to their physician due to AR in the current calendar year, emphasizing the burden uncontrolled disease has on healthcare systems. By providing rapid and effective AR symptom relief, MP-AzeFlu has the potential to reduce both the clinical and economic impact of the disease. The study also showed the very low usage of allergen immunotherapy (AIT) for moderate-to-severe AR ( $n=30,11.9 \%)$, highlighting the need to increase physician knowledge of AIT disease-modifying benefits.

The effectiveness of MP-AzeFlu observed in this real-life setting was better than its efficacy reported in RCTs, emphasizing the importance of real-life data to complement that obtained in a controlled clinical setting. In the RCT setting, patients with moderate-to-severe SAR treated with MP-AzeFlu for 2 weeks experienced twice the nasal and ocular symptom relief than those treated with an INS; one in six of them achieved complete or near-to-complete symptom relief and about a week faster than FP monotherapy ${ }^{(23)}$. The responder rates in the current real-life study were higher than that. Patients treated with MP-AzeFlu experienced a $63.6 \mathrm{~mm}$ reduction in VAS score, almost three times the reduction required for clinical relevance ${ }^{(35)}$. Furthermore, 8 out of 10 patients treated with MP-AzeFlu in routine clinical practice crossed the well-controlled zone by end of treatment (i.e. VAS score $\leq 38 \mathrm{~mm}$ ).

The results of this study in Romania showed better symptom control with MP-AzeFlu compared to other real-life studies with MP-AzeFlu conducted in Scandinavia ${ }^{(32-34)}$ and Germany ${ }^{(31)}$. For example, the mean change from baseline in VAS score in this study was $63.6 \mathrm{~mm}$, higher than that reported in Sweden (36.1 $\mathrm{mm})^{(32)}$, Denmark $(38.8 \mathrm{~mm})^{(33)}$, Norway $(30.8 \mathrm{~mm})^{(34)}$ and Germany $(54.1 \mathrm{~mm})^{(31)}$. Patient perception of AR control was, however, consistent amongst the countries with $95.2 \%$ of Romanian AR patients feeling their AR was well- or partly controlled at Day 3, compared with $84.0 \%, 85.6 \%, 88.1 \%$ and $94.3 \%$ of patients in Sweden, Denmark, Norway and Germany, respectively ${ }^{(31-34)}$. Irrespective of country, MP-AzeFlu was well-tolerated and provided rapid, sustained and clinically relevant AR control.

The main limitations of this non-interventional, observational study were its open-label design and the lack of placebo or active comparator. A head-to-head comparison between patientdefined control in the current study and the arbitrary cut-offs applied in RCTs would also have been welcomed, but since reflective total nasal symptom score is not routinely assessed in real-life, this was not possible.

\section{Conclusion}

MP-AzeFlu is a well-tolerated treatment that provides effective, fast and sustained symptom control in a real-world setting in Romanian AR patients with uncontrolled disease despite monoand multiple-therapy usage and multiple doctor visits. The benefit was consistent irrespective of disease severity, phenotype or patient age and supports MP-AzeFlu's position as the drug of choice for the treatment of uncontrolled moderate-to-severe AR 
in real-life.

\section{Acknowledgements}

Medical writing assistance in the preparation of this manuscript was provided by Dr Ruth B Murray and Dr Winnie McFadzean, Medscript Ltd, and Dr Roger Hill, Ashfield Healthcare Communications, funded by MEDA Pharma GmbH \& Co. KG. We thank Dr DunTung Nguyen and Dr Hans-Christian Kuhl for critical assessment of the data and manuscript review. This study was funded by Meda.

\section{Authorship contribution}

All authors contributed to interpretation of data, drafting of the article and revising it critically, and provided final approval of the version to be published.

\section{Conflict of interest}

PML declares that Meda has previously sponsored his attendance at scientific congress. All other authors declare no conflicts of interest.

\section{References}

1. Agache I, Deleanu D, Khaltaev N, Bousquet J. [Allergic rhinitis and its impact upon asthma--update (ARIA 2008). Romanian perspective]. Pneumologia. 2009;58:255-8.

2. Bjorksten B, Dumitrascu D, Foucard T, et al. Prevalence of childhood asthma, rhinitis and eczema in Scandinavia and Eastern Europe. Eur Respir J. 1998;12:432-7.

3. Chereches-Panta P, C S, Dumitrescu D, et al. Epidemiological survey 6 years apart: increased prevalence of asthma and other allergic diseases in schoolchildren aged 13-14 years in cluj-napoca, romania (based on isaac questionnaire). Maedica (Buchar). 2011;6:10-6.

4. Aït-Khaled N, Pearce N, Anderson HR, Ellwood P, Montefort S, Shah J; ISAAC Phase Three Study Group. Global map of the prevalence of symptoms of rhinoconjunctivitis in children: The International Study of Asthma and Allergies in Childhood (ISAAC) Phase Three. Allergy. 2009;64:123-48.

5. Valovirta E, Myrseth SE, Palkonen S. The voice of the patients: allergic rhinitis is not a trivial disease. Curr Opin Allergy Clin Immunol. 2008;8:1-9.

6. Bousquet PJ, Demoly P, Devillier P, Mesbah K, Bousquet J. Impact of allergic rhinitis symptoms on quality of life in primary care. Int Arch Allergy Immunol. 2013;160:393 400.

7. Colas C, Galera H, Anibarro B, et al. Disease severity impairs sleep quality in allergic rhinitis (The SOMNIAAR study). Clin Exp Allergy. 2012;42:1080-7

8. Marshall PS, O'Hara C, Steinberg P. Effects of seasonal allergic rhinitis on fatigue levels and mood. Psychosom Med. 2002;64:68491

9. Walker S, Khan-Wasti S, Fletcher M, Cullinan P, Harris J, Sheikh A. Seasonal allergic rhinitis is associated with a detrimental effect on examination performance in United Kingdom teenagers: case-control study. Allergy Clin Immunol. 2007;120:381-7.

10. Price D, Scadding G, Ryan D, et al. The hidden burden of adult allergic rhinitis: UK healthcare resource utilisation survey. Clin Transl Allergy. 2015;5:39.

11. Cardell LO, Olsson P, Andersson M, et al.
TOTALL: high cost of allergic rhinitis-a national Swedish population-based ques tionnaire study. NPJ Prim Care Respir Med. 2016;26:15082

12. Bousquet J, Khaltaev N, Cruz AA, et al. Allergic Rhinitis and its Impact on Asthma (ARIA) 2008 update (in collaboration with the World Health Organization, GA(2)LEN and AllerGen). Allergy. 2008;63 Suppl 86:8160.

13. Brozek JL, Bousquet J, Baena-Cagnani CE et al. Allergic Rhinitis and its Impact on Asthma (ARIA) guidelines: 2010 revision. J Allergy Clin Immunol. 2010;126:466-76.

14. Ciprandi G, Incorvaia C, Scurati S, et al. Patient-related factors in rhinitis and asthma: the satisfaction with allergy treatment survey. Curr Med Res Opin. 2011;27:1005-11.

15. Navarro A, Valero A, Rosales MJ, Mullol J. Clinical use of oral antihistamines and intranasal corticosteroids in patients with allergic rhinitis. J Investig Allergol Clin Immunol. 2011;21:363-9.

16. Price DB, Scadding G, Bachert C, et al. UK prescribing practices as proxy markers of unmet need in allergic rhinitis: a retrospective observational study. NPJ Prim Care Respir Med. 2016;26:16033.

17. Anolik R, Mometasone Furoate Nasal Spray With Loratadine Study G. Clinical benefits of combination treatment with mometasone furoate nasal spray and loratadine vs monotherapy with mometasone furoate in the treatment of seasonal allergic rhinitis. Ann Allergy Asthma Immunol. 2008;100:264-71.

18. Esteitie R, deTineo M, Naclerio RM, Baroody FM. Effect of the addition of montelukast to fluticasone propionate for the treatment of perennial allergic rhinitis. Ann Allergy Asthma Immunol. 2010;105:155-61.

19. Bousquet J, Kuh D, Bewick M et al. Operational Definition of Active and Healthy Ageing (AHA): A Conceptual Framework. J Nutr Health Aging. 2015;19:955-60.

20. Bousquet J, Schunemann HJ, Fonseca J, et al. MACVIA-ARIA Sentinel Network for allergic rhinitis (MASK-rhinitis): the new generation guideline implementation. Allergy. 2015;70:1372-92.

21. Bousquet J, Schunemann $H$, Arnavielhe S, et al. MACVIA clinical decision algorithm in allergic rhinitis in adolescents and adults. J Allergy Clin Immunol. 2016;138:367-74.

22. Bousquet J, Bachert C, Price D, Acaster S, Demoly P. Assessing allergic rhinitis symptom control using a simple visual analogue scale: the digital solution. Allergy. 2014;69.

23. Meltzer E, Ratner P, Bachert C, et al. Clinically relevant effect of a new intranasal therapy (MP29-02) in allergic rhinitis assessed by responder analysis. Int Arch Allergy Immunol. 2013;161:369-77.

24. Price D, Shah S, Bhatia S, et al. A new therapy (MP29-02) is effective for the long-term treatment of chronic rhinitis. J Investig Allergol Clin Immunol. 2013;23:495-503.

25. Costa DJ, Amouyal M, Lambert P, et al. How representative are clinical study patients with allergic rhinitis in primary care? J Allergy Clin Immunol. 2011;127:920-6 e1.

26. Price D, Smith $P$, Hellings $P$, et al. Current controversies and challenges in allergic rhinitis management. Expert Rev Clin Immunol. 2015;11:1205-17.

27. Dymista summary of product characteristics for Norway. https://www.medicines.org. uk/emc/medicine/27579 2014. Accessed.

28. Klimek L, Bachert, C., Stjarne, P., Dollner, R., Larsen, P., Haahr, P., Agache, I., Scadding, G., Price, D. MP-AzeFlu provides rapid and effective allergic rhinitis control in real-life: a pan-European study. Allergy Asthma Proc. 2016 Sep:37(5):376-86.

29. Stull DE, Roberts L, Frank L, Heithoff $K$. Relationship of nasal congestion with sleep, mood, and productivity. Curr Med Res Opin. 2007:23:811-9.

30. Canonica GW, Bousquet J, Mullol J, Scadding GK, Virchow JC. A survey of the burden of allergic rhinitis in Europe. Allergy. 2007:62 Suppl 85:17-25.

31. Klimek L, Bachert C, Mosges R, et al. Effectiveness of MP29-02 for the treatment of allergic rhinitis in real-life: results from a noninterventional study. Allergy Asthma Proc. 2015;36:40-7.

32. Stjarne $P$, Strand $V$, Theman K, Kuhl H, Ehngage A. Real-life effectiveness of a new allergic rhinitis thrapy (MP29-02) in Sweden. Allergy. 2015;70.

33. Haahr $\mathrm{P}$, Jacobsen C, Blegvad S, Christensen 
M, Kuhl $H$, Nielsen K. Real life effectiveness of a new allergic rhinitis therapy (MP29-02) in Denmark. Allergy. 2015;70.

34. Dollner R, Lorentz Larsen P, Sheyauldeen S, Kuhl H, Steinsvag S. Real-life effectiveness of a new allergic rhinitis therapy (MP29-02) in Norway. Allergy. 2015;70.

35. Demoly P, Bousquet PJ, Mesbah K, Bousquet J, Devillier P. Visual analogue scale in patients treated for allergic rhinitis: an observational prospective study in primary care: asthma and rhinitis. Clin Exp Allergy 2013;43:881-8.
Prof loana Agache

2A Pictor lon Andreescu

Brasov

Romania, 500051

Tel: +40727849321

Fax:+40 268516689

E-mail: ibrumaru@unitbv.ro 